\title{
Social Recipe Recommendation to Reduce Food Waste
}

Fulya Yalvaç

Eindhoven University of

Technology, Department of

Industrial Design

Eindhoven The Netherlands

f.yalvac@tue.nl

Veranika Lim

Eindhoven University of

Technology, Department of

Industrial Design

Eindhoven The Netherlands

v.lim@tue.nl

Jun $\mathrm{Hu}$

Eindhoven University of

Technology, Department of

Industrial Design

Eindhoven The Netherlands

j.hu@tue.nl

\author{
Mathias Funk \\ Eindhoven University of \\ Technology, Department of \\ Industrial Design \\ Eindhoven The Netherlands \\ m.funk@tue.nl \\ Matthias Rauterberg \\ Eindhoven University of \\ Technology, Department of \\ Industrial Design \\ Eindhoven The Netherlands \\ g.w.m.rauterberg@tue.nl
}

Permission to make digital or hard copies of part or all of this work for personal or classroom use is granted without fee provided that copies are not made or distributed for profit or commercial advantage and that copies bear this notice and the full citation on the first page. Copyrights for third-party components of this work must be honored. For all other uses, contact the owner/author(s). Copyright is held by the author/owner(s). CHI 2014, April 26-May 1, 2014, Toronto, Ontario, Canada.

ACM 978-1-4503-2474-8/14/04.

http://dx.doi.org/10.1145/2559206.2581226

\begin{abstract}
Little attention has been given to food waste prevention in households by changing consumers' behaviors. In this paper, we present a social recipe recommender which is a mobile application being developed to reduce food waste in households by recommending recipes to a group of connected people. The application will allow the logging of food and waste related daily practices and will persuade a group of users to share their food by recommending recipes based on available ingredients within this group.

The method of data collection, persuasion and the experiments are described through this paper.
\end{abstract}

\section{Author Keywords}

Recommendation Systems; Persuasive Technology; Food Waste; Mobile Computing

\section{ACM Classification Keywords}

H.5.m [Information interfaces and presentation]:

Miscellaneous.

\section{Introduction}

Food waste is becoming a central issue of the twenty-first century. A total of one third up to one half of all produced food is wasted according to the estimates [14]. In developed countries, most of this food waste is generated in the latest stage of the supply chain, the 


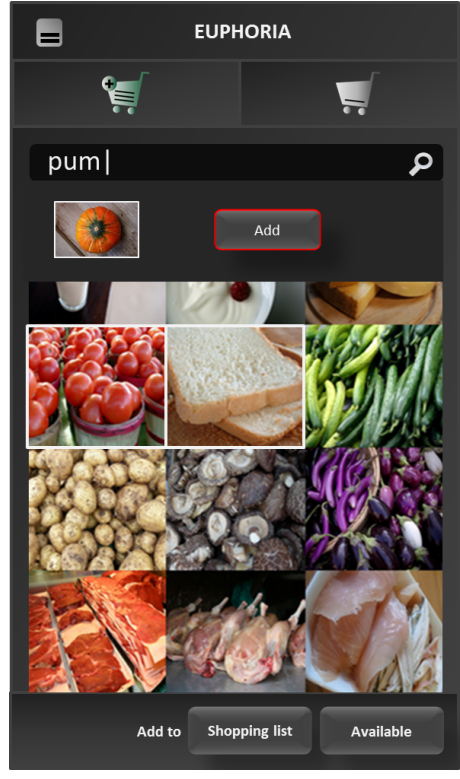

Figure 1: Ingredient selection consumer $[13,14]$. In the UK, for instance, about 7 million tons of food is thrown away from homes each year [14]. This waste does not only contain edible food losses but also loss of energy, land and water [14]. Changing consumers' behaviors is therefore highly important. Despite the increasing interest in using technology to encourage ecologically sustainable living, food waste is still one of the topics that needs further exploration at the consumer level.

So far, $\mathrm{HCl}$ and persuasive technology studies aiming at food waste reduction in households mainly focused on intelligent fridge applications that can sense wasteful behaviors [19], social normative persuasion studies [6, 23] or food waste diary concepts [10]. In these studies, the technology tries to track waste data and provides behavioral feedback in order to motivate reflections. So far, giving users a specific direction on how to decrease resource consumption is not commonly done in persuasive sustainability research [4]. To promote more sustainable or healthy life styles, social media is also used as a major tool to engage social influence in a positive way $[8,17]$. In these studies, online social networks are facilitated successfully to expose social norms, which resulted in a more collective behavior change. Encouraging social dinners through social platforms have also been studied to enhance relationships among community or to enable sharing cooking experiences [12, 15, 22]. However, providing a social platform with a specific goal of reducing food waste in households is underexplored to our knowledge. This research is focused on the question of how recommendation systems could be used as a tool to explore innovative ways of encouraging social collaboration to reduce food waste. To answer this question, we are developing EUPHORIA, a mobile application that can suggest recipes to a group of friends by combining the available ingredients from different households, based on the preferences of each person in the group. Instead of accounting social norms, this study focuses on changing consumers' behaviors by using a recommendation system. In the rest of the paper, we will explain the design of the mobile application, how the recommendation process will work and the measures used in this study.

\section{Data Collection}

User Interaction Design

EUPHORIA is a mobile application that can be divided into two phases which are data tracking and recipe recommendation. Data tracking is for collecting data related to what kind of food users buy, consume and waste and the reasons of the waste. Recipe recommendation will be applied later when the system has data to construct user profiles and to apply recommendation algorithms.

For data tracking (Phase 1), the users search and select the items they buy or plan to buy through the interface shown in Figure 1. In order to enhance the user experience, the main page shows the mostly chosen items with selectable image buttons. Users are able to search the other ingredients by typing their names. As soon as three letters are typed, a list of ingredients containing the written word is shown. The user will complete the selection by pressing either to the shopping list or to the available list button. The shopping list consists of the items users plan to buy and the available list is composed of ingredients they already have (see Figure 2). As soon as an item in the shopping list is confirmed, it will be appended in the available list. We expect the shopping lists to be one of the reasons for people to use the application that can increase the logging frequency. After users have selected the ingredients, they can indicate the amount of each item to complete the logging (see 


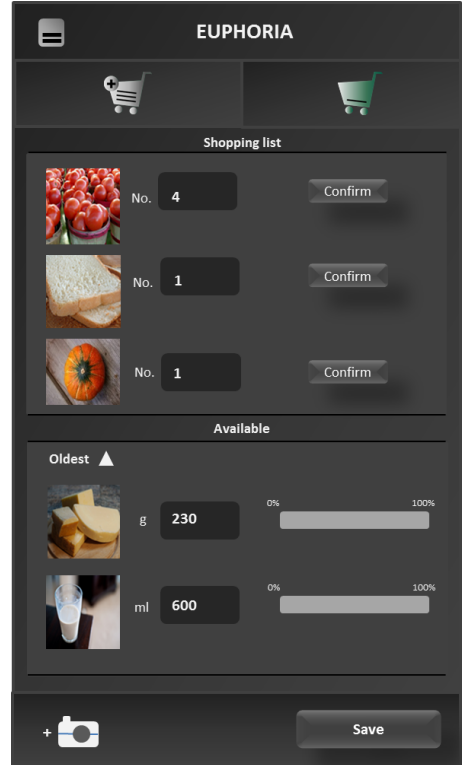

Figure 2: Sample user food list

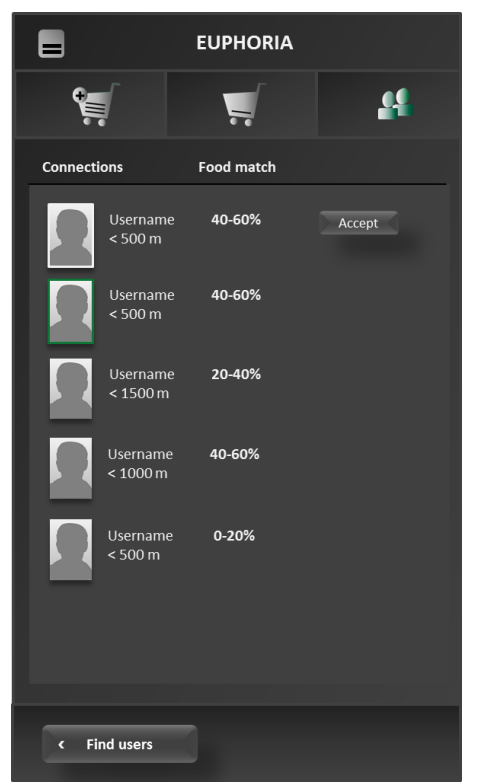

Figure 2). The users are reminded one time per day to enter the percentage of the ingredients they have used. This is an important trigger of the system, which is described as one of the key attributes of persuasive design in [7]

Users are able to delete an item as soon as it has been consumed or thrown away. When an item is deleted before all of it is consumed, the system records it as a wasted item and the user is directed to enter the reason with mobile notifications. Mobile notifications are found to have a positive effect on increasing the manual logging of food intake more than five times compared to the case with no notifications [2]. Although users are not obliged to give a reason of their food waste, the same strategy might be integrated into this case as well to increase the frequency of logging.

In Phase 2, users will be able to connect with other people to share their food related information (see Figure 3).

They will be able to send invitations to connect with other users and list them according to their distance and food match. Distance is the real physical distance measured with the address information of users. Food match shows the similarity of one's shopping list with the other user's available list. This means if a user has the ingredients (i.e. having them in the available list) another user wants to buy (i.e. user has added them into the shopping list), the system will calculate their food match as $100 \%$ or vice versa. Users will be able to notify their friends to initiate food sharing through the application. We think the possibility of sharing food physically can both increase the frequency of food logging and decrease the amount of forgotten food. The system will also recommend recipes to a group of people who are connected with each other based on their available ingredients and food preferences as shown in Figure 4. The recipe recommendations are displayed with the amount of savings user will make if the item is selected. It is highly important to state that displaying savings does not serve as a motivation for people to accept the recommendation. As stated in [20], extrinsic motivations such as money cannot be effective solutions for changing complicated behaviors and besides they can even be de-motivators. In fact, the amount displayed is not the actual gain of a person because in reality, users are sharing food which does not serve for making profit. Thus, this is more about making the consequences of food waste visible to the consumers, which was found to be one of the solutions to promote sustainable behavior [11, 18] and emphasized by [10]. As soon as all users in the group confirm the

recommendation, they will be notified to enjoy their dinner together.

Implementation Details

The application is developed by using an hybrid approach with PhoneGap as a connecter with native functions and JQueryMobile for user interface design together with web technologies such as Javascript, HTML and CSS. Play framework for JAVA is used to implement the server side and client-server communication. Deployment for different mobile platforms is done with PhoneGap Cloud Build facility. The icons for ingredients are taken from image-net database[1].

\section{Recommendation Approach}

Recommendation systems have been widely used in e-commerce and entertainment in recent years in addition to the studies in data mining research[21]. Most of these studies focused on improving the efficiency of the rating prediction algorithm [5]. Leaving out user experiments using interactive interfaces led to the problem of a gap

Figure 3: List of users 


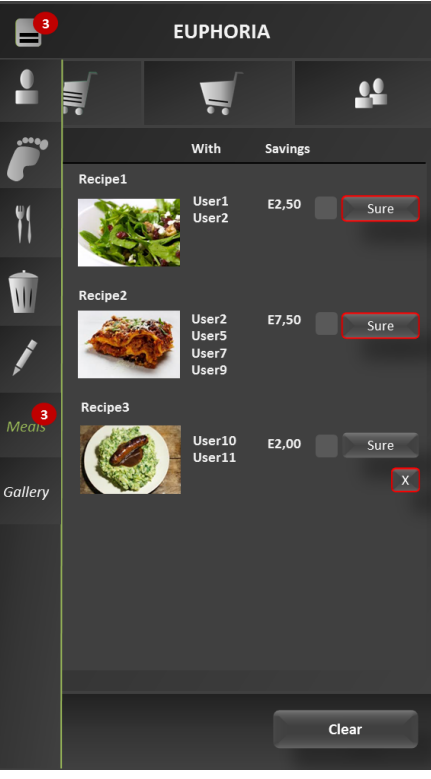

Figure 4: Recommendations between the real success of the recommendation system and measured efficiency of the prediction algorithm $[3,16]$. Instead of focusing on improving the prediction accuracy, this project concerns more about the design of the user experience and its implications to improve user satisfaction and to meet the system needs. The satisfaction of users is defined as accepting suggested recipes and the system need is described as reducing the total food waste. The system will identify the recipe that is both based on available ingredients and satisfaction among users in the group. To achieve this, a recommendation approach described below will be followed.

The data retrieved with food logging will be used to find what ingredients users like, which will be used to identify the user-ingredient rating matrix for the recipe recommendation. This matrix is the measurement of users' items preferences [21]. The bigger the rating(r) in the matrix for corresponding user(u) and item(i), the more the user u likes item i. In general, recommendation systems have two ways of data collection which are explicit and implicit [21]. EUPHORIA will use implicit data collection by using purchasing frequency as a rating for ingredients. The frequency of purchasing is not useful to understand user preferences for the ingredients like rice or grains, which are purchased in bulk but consumed very often. However these products are not wasted too much due to their long term durability. Therefore we will not concentrate on improving efficient consumption of dry products like rice and grains as a first goal. In addition to that, the link between ingredient and recipe needs to be described. In [9], Freyne et al. transferred the ratings gathered from ingredients to their associated recipes by taking the average ratings of its ingredients. The same method will be applied to EUPHORIA. The system will recommend items similar to users' previous choices, which is called as content based recommendation algorithm [21]. Item similarity is the measurement of how much a recipe is similar to another one, which will be calculated with the correspondence of their ingredients. Additionally, by converting consumption information to user-ingredient ratings, a user-user similarity matrix will be extracted. This matrix will be used by the recommender to suggest recipes based on user similarity, which is defined as collaborative filtering algorithm [21]. Similarity of users is expressed in two different ways: The percentage of how similar one user's food preferences are with the other user's choices and the percentage of the common ingredients in one user's shopping list with the other one's available list.

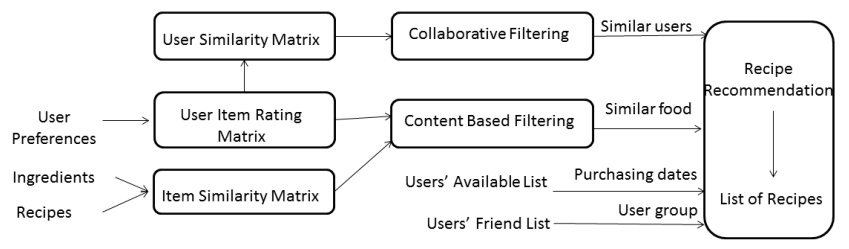

Figure 5: Recommendation Approach

The recommendation process will first sort the items of a group of friends according to the expiration dates. Then it will identify an item that should be consumed sooner than the other ingredients for each user by checking average endurance periods, date of purchase and combine it within a recipe. The output of the recommendation is a list of recipes for groups ordered by their chance of being accepted (See Figure 5).

\section{Procedure}

The user studies will be held in three parts: interviews, testing data collection (Phase 1) and testing the recipe 
recommendation (Phase 2). In the first part, participants will be interviewed about their weekly food waste. They will be asked to collect receipts for food usage detection. This will last for one month. In the second part, participants will be asked to log their food practices with EUPHORIA. The data will be compared with the output of the interviews to form a baseline. This will last for 2 months. The third part of the experiments will be for testing the effect of recommendation. In this part, participants will continue to log their data and they will be suggested with recipes according to the specifications described in the previous chapter. The validity of the system will be tested with quantitative data which is the amount of waste occurred during the experiment process and the acceptance rate of the recipes. The experiments will last six months. Students and professionals in The Netherlands living together or in a complex with shared kitchens will be recruited. Participants are expected to have a smart phone and download the application suitable with their platform.

\section{Discussions}

The aim of this project is to design a persuasive technology to reduce food waste in households by creating an interactive and social platform for users. Long term experiments and analysis are required not only to change behaviors of people but also to measure this change. In addition to that, recommendation systems need big amount of data to work properly. Long term logging of data is therefore highly important. Although data logging is not our main focus in this study, we are aware that it should not be a burden for the users. Therefore we try to design the user interaction with the least intervention possible and engage users with functions that can be beneficial for them such as using the application for creating shopping lists, being able to see ingredients of other users and sharing food. By including users in the earlier phase of the design, we hope to overcome the possible problems related to the engagement with the tool.

EUPHORIA provides a social platform for people to share food as well as to appreciate it together. The idea is originated from the increasing interest in internet of things in our daily lives. We believe that it is likely to have smart environments that can log food activities in households in the near future. When the technology is smart enough to track exactly the types and amounts of ingredients consumers have, EUPHORIA can play an important role on how to use this data to reduce food waste. We propose a recipe recommendation system based on available ingredients as the current solution for a positive impact towards sustainable behavior. We think this project contributes to sustainable $\mathrm{HCl}$ and persuasive technology studies as well as providing a new application area for the design and testing of recommendation systems.

\section{Acknowledgements}

This work is supported in part by the Erasmus Mundus Joint Doctorate in Interactive and Cognitive Environments (ICE), which is funded by the EACEA Agency of the European Commission under EMJD ICE FPA n2010-0012. The authors would like to thank Andreu Catala from the Technical University of Catalonia and Lucio Marcenaro and Carlo Regazzoni from the Università degli Studi di Genova for their feedback and suggestions.

\section{References}

[1] Image-net database. http://www.image-net.org/. Accessed: 2013-10-21.

[2] Bentley, F., and Tollmar, K. The Power of Mobile Notifications to Increase Wellbeing Logging Behavior. CHI (2013). 
[3] Bobadilla, J., Ortega, F., Hernando, a., and Gutiérrez, a. Recommender Systems Survey. Knowledge-Based Systems 46 (Apr. 2013), 109-132.

[4] Brynjarsdóttir, H., Hå kansson, M., Pierce, J., Baumer, E. P. S., Disalvo, C., and Sengers, P. Sustainably Unpersuaded: How Persuasion Narrows Our Vision of Sustainability. CHI12, May 510, 2012, Austin, Texas, USA (2012), 947-956.

[5] Burke, R. Hybrid Recommender Systems : Survey and Experiments . 1-29.

[6] Farr-Wharton, G., Foth, M., and Choi, J. H.-j. EatChaFood: Challenging Technology Design to Slice Food Waste Production. UbiComp13 (2013), 559-562.

[7] Fogg, B. A behavior model for persuasive design. Proceedings of the 4th International Conference on Persuasive Technology - Persuasive '09 (2009), 1.

[8] Foster, D., and Lawson, S. Liking Persuasion: Case studies in Social Media for Behaviour Change. 1-4

[9] Freyne, J., and Berkovsky, S. Recommending Food: Reasoning on Recipes and Ingredients. 381-386.

[10] Ganglbauer, E., Fitzpatrick, G., and Molzer, G. Creating Visibility: Understanding the Design Space for Food Waste. MUM12 (2012), 0-9.

[11] Griskevicius, V., Cantú, S. M., and Vugt, M. V. The Evolutionary Bases for Sustainable Behavior: Implications for Marketing, Policy, and Social Entrepreneurship. Journal of Public Policy \& Marketing 31, 1 (May 2012), 115-128.

[12] Gross, S., Toombs, A., Wain, J., and Walorski, K. Foodmunity : Designing community interactions over food (2011)

[13] Gustavsson, J., Cederberg, C., and Sonesson, U. Global Food Losses and Food Waste. Food and Agriculture Organization of the United Nations (2011).
[14] Institution of Mechanical Engineers. Global Food Waste Not, Want Not.

[15] Kanai, H., and Kitahara, K. A Menu-planning Support System to Facilitate Communication among Neighbors. CSCW (2011)

[16] Konstan, J. a., and Riedl, J. Recommender systems: from algorithms to user experience. User Modeling and User-Adapted Interaction 22, 1-2 (Mar. 2012), 101-123.

[17] Linehan, C., Leeman, T., Borrowdale, C., and Lawson, S. Crowd Saucing : Social Technology for Encouraging Healthier Eating. 53-57.

[18] Manning, C. The Psychology of Sustainable Behavior.

[19] Murata, S., Kagatsume, S., Taguchi, H., and Fujinami, K. PerFridge: An Augmented Refrigerator That Detects and Presents Wasteful Usage for Eco-Persuasion. 2012 IEEE 15th International Conference on Computational Science and Engineering (Dec. 2012), 367-374.

[20] Pink, D. H. The surprising truth about what motivates us. Soundview Executive Book Summaries, 2010.

[21] Ricci, F., Rokach, L., Shapira, B., and Kantor, P. B. Recommender Systems Handbook. Springer US, Boston, MA, 2011.

[22] Svensson, M., Höök, K., and Cöster, R. Designing and Evaluating Kalas : a Social Navigation System for Food Recipes. 1-32.

[23] Thieme, A., Comber, R., Miebach, J., Weeden, J., Krämer, N., Lawson, S., and Olivier, P. We've Bin Watching You-Designing for Reflection and Social Persuasion to Promote Sustainable Lifestyles. $\mathrm{CHI}$ 2012, May 510, 2012, Austin, Texas, USA (2012), 2337-2346 